\title{
Aranhas da família Theridiosomatidae: espécie nova e novas ocorrências no Brasil
}

\author{
Everton N. L. Rodrigues ${ }^{1,3}$ \& Ricardo Ott ${ }^{2}$
}

\begin{abstract}
1. Programa de Pós-Graduação em Biologia Animal, Departamento de Zoologia, Instituto de Biociências, Universidade Federal do Rio Grande do Sul. Av. Bento Gonçalves, 9500, Bloco IV, Prédio 43435, 91501-970 Porto Alegre, RS, Brasil. (enlrodrigues@ yahoo.com.br) 2. Museu de Ciências Naturais, Fundação Zoobotânica do Rio Grande do Sul, Rua Dr. Salvador França, 1427, 90690-000 Porto Alegre, RS, Brasil. (rott@fzb.rs.gov.br)

3. Bolsista CNPq.
\end{abstract}

ABSTRACT. Spiders of family Theridiosomatidae: a new species and new records for Brazil. Theridiosoma caaguara sp. nov. from Rio Grande do Sul state, Brazil, is described and illustrated. Baalzebub baubo Coddington, 1986 is recorded for the first time for Brazil and the known geographic distribution of Epilineutes globosus (O. P.-Cambridge, 1896), Naatlo splendida (Taczanowski, 1873) and Ogulnius obtectus O. P.-Cambridge, 1882 is extended.

KEYWORDS. Araneae, Theridiosoma, Neotropical, taxonomy, distribution.

RESUMO. Theridiosoma caaguara sp. nov. do Estado do Rio Grande do Sul, Brasil, é descrita e ilustrada. Baalzebub baubo Coddington, 1986 é registrada pela primeira vez para o Brasil, ampliando-se a distribuição conhecida de Epilineutes globosus (O. P.-Cambridge, 1896), Naatlo splendida (Taczanowski, 1873) e Ogulnius obtectus O. P.-Cambridge, 1882.

PALAVRAS-CHAVE. Araneae, Theridiosoma, Neotropical, taxonomia, distribuição.

A família Theridiosomatidae é composta por aranhas caracterizadas, principalmente, pelo esterno largamente truncado na região posterior e com glândulas em depressões localizadas na promargem, adjacentes ao lábio, clípeo elevado e longas tricobótrias tibiais (CodDington, 1986). Constroem teias orbiculares complexas (ARCHER, 1953) e preferem locais escuros (CODDINGTON, 1986).

Dos 12 gêneros atualmente incluídos na família Theridiosomatidae, sete ocorrem no Brasil (PLATNICK, 2005): Chthonos Coddington, 1986; Epilineutes Coddington, 1986; Naatlo Coddington, 1986; Ogulnius O. P.-Cambridge, 1882; Plato Coddington, 1986; Theridiosoma O. P.-Cambridge, 1879 e Wendilgarda Keyserling, 1886.

Coddington (1986) estudou muitas espécies neotropicais, no entanto, nenhum material de coleções brasileiras foi examinado. Ao examinar a coleção aracnológica do Museu de Ciências Naturais, Fundação Zoobotânica do Rio Grande do Sul (MCN; E. H. Buckup) foi possível detectar uma espécie nova do gênero Theridiosoma, além de registrar pela primeira vez para o Brasil Baalzebub baubo Coddington, 1986. Para Epilineutes globosus (O. P.-Cambridge, 1886), Naatlo splendida (Taczanowski, 1879) e Ogulnius obtectus O. P.-Cambridge, 1882 são fornecidas novas ocorrências, ampliando a área de distribuição conhecida destas espécies no Brasil.

Descrições e a terminologia do palpo seguem Coddington (1986; 1990). Todas as medidas são apresentadas em milímetros (mm).

\section{Theridiosoma caaguara sp. nov.} (Figs. 1-6)

Material-tipo. Holótipo ơ', BRASIL, Rio Grande do Sul:
São Francisco de Paula (Centro de Pesquisas e Conservação da Natureza Pró-Mata), 11.X.2001, R. Ott col., pitfall trap (MCN 39018).

Etimologia. O nome específico refere-se aos índios da tribo caaguara, primeiros habitantes na localidade-tipo.

Diagnose. O macho de Theridiosoma caaguara (Figs. 1-6) possui o palpo similar ao de T. gemmosum (L. Koch, 1877) (CodDingTon, 1986, figs. 134-137, 146-149) pela presença de dentículos na margem do tégulo abaixo da base do condutor (Fig. 4), mas distingue-se pela forma do condutor alargada e mais distante do címbio, apófise média sobre o condutor (Fig. 4) terminando arredondada, com uma pequena dobra, apófise embólica esclerotinizada com região distal afilada (Figs. 4, 5), não projetada como em T. goodnightorum Archer, 1953 (CoDDINGTON, 1986, figs. 130, 131) e $T$. chiripa Rodrigues \& Ott, 2005 (Rodrigues \& OTt, 2005, fig. 4).

Descrição. Holótipo ơ Comprimento total 1,53. Carapaça, comprimento 0,75 ; largura 0,73 ; região cefálica 0,56. Esterno, comprimento 0,37; largura 0,40. Clípeo, altura 0,18. Abdômen, comprimento 0,97; largura 0,93; altura 1,10. Fórmula das pernas 1,2,4,3. Comprimento 1/2/ $3 / 4$ : fêmures $0,85 / 0,75 / 0,37 / 0,50$; patelas $0,30 / 0,27 / 0,20 /$ 0,22 ; tíbias $0,72 / 0,57 / 0,22 / 0,42$; metatarsos $0,57 / 0,50 / 0,30 /$ 0,47 ; tarsos $0,35 / 0,30 / 0,22 / 0,22$; total 2,79/2,39/1,31/1,83. Carapaça amarelo-clara, área ocular castanho-escura. Quelíceras, lábio e enditos amarelo-claros. Esterno castanho-amarelado com bordas enegrecidas (Fig. 2). Pernas amarelo-claras, exceto coxas com bordas distais escuras e tarsos castanho-amarelados. Abdômen dorsalmente amarelo-claro, laterais e região posterior 
castanhas, na região mediana com uma linha castanhoescura transversal e outra longitudinal em forma de cruz (Figs. 1, 3). Ventre do abdômen castanho-escuro, com laterais amarelo-claras. Olhos heterogêneos, OMP maiores que os demais, OLA mais desenvolvidos que os OLP. Palpo com tégulo desenvolvido (Figs. 4, 6), paracímbio rudimentar, assim como o subtégulo, ápice do címbio curvo (Fig. 6), êmbolo curto e pouco desenvolvido (Figs. 4, 5).

Distribuição. Sul do Brasil (Rio Grande do Sul).

\section{Baalzebub baubo Coddington, 1986}

Baalzebub baubo Coddington, 1986:72, figs. 161, 162, 168, 179-187.

Material examinado. BRASIL, Santa Catarina: Porto Belo (Praia de Bombas), O', 14.II.1990, A. D. Brescovit col. (MCN 19462).

Distribuição. Costa Rica (Heredia), Panamá (Panamá) e Brasil (Santa Catarina).
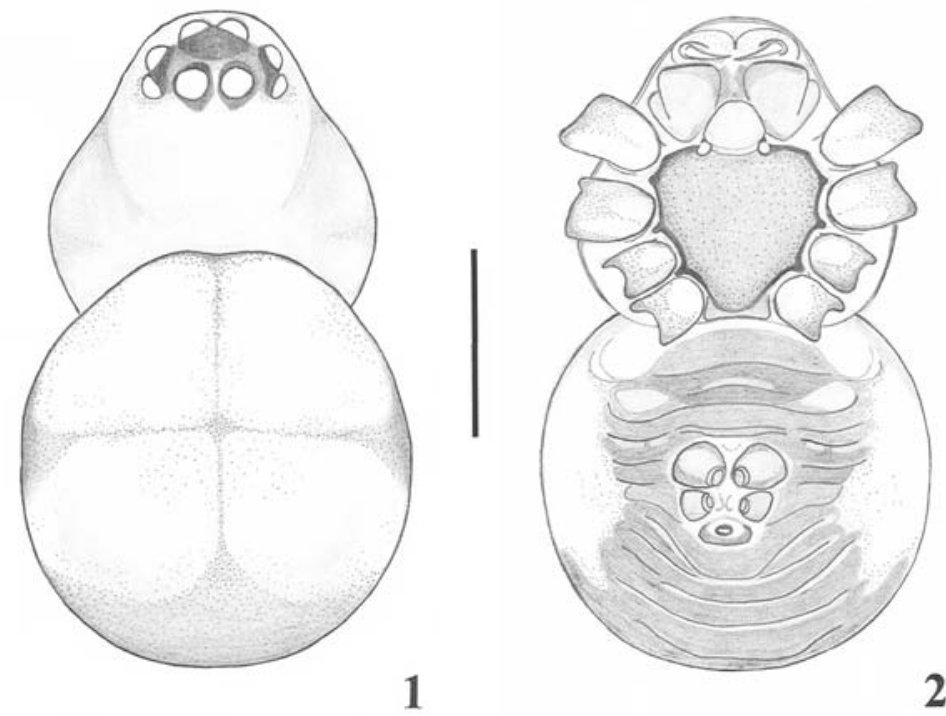
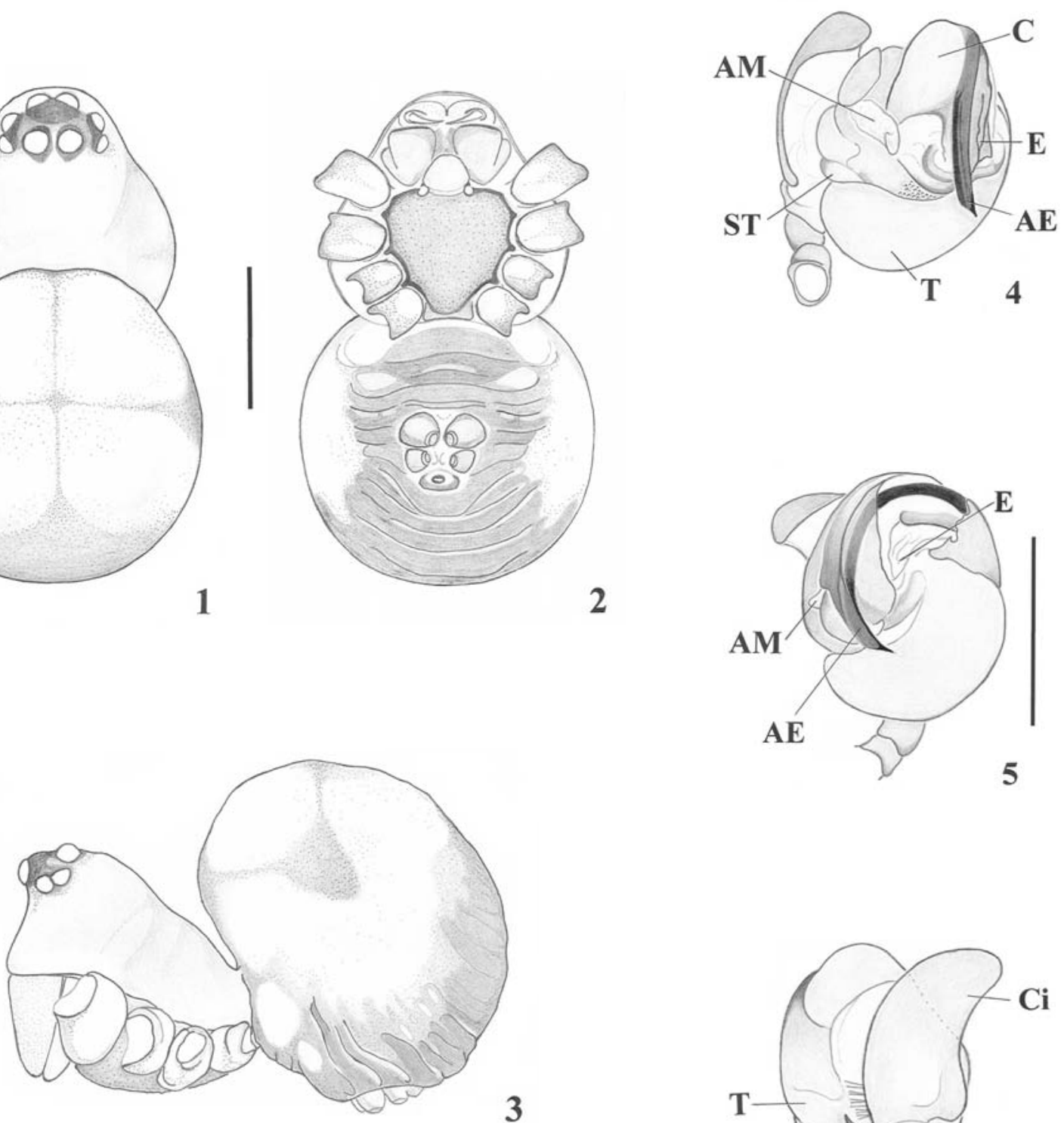

3

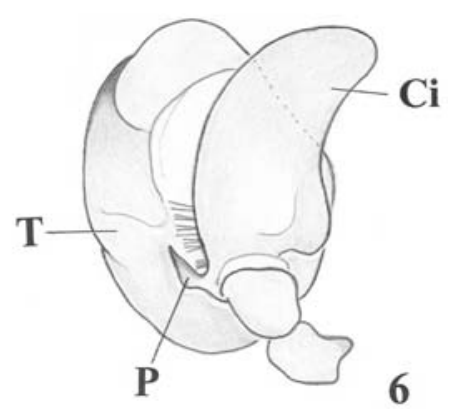

Figs. 1-6. Theridiosoma caaguara sp. nov.: 1-3, macho: 1, dorsal; 2, ventral; 3, lateral. 4-6, palpo: 4, ventral; 5, retrolateral; 6, dorsal (AE, apófise embólica; AM, apófise média; C, condutor; Ci, címbio; E, êmbolo; P, paracímbio; ST, subtégulo; T, tégulo). Barras, 0,5 $\mathrm{mm}$. 


\section{Epilineutes globosus (O. P.-Cambridge, 1896)}

Andasta globosa O. P.-CAMBridge, 1896:192, pr. 24, figs. 5, 6. Theridiosoma globosum; F. O. Pickard-CAMBridge, 1902:414; Simon, 1903:1007; Roewer, 1942:970; ARCHER, 1953:11, fig. 16; BonNet, 1959: 4434.

Epilineutes globosus; CoDdington, 1986:79, figs. 169-178, 188-195.

Material examinado. BRASIL, Rio Grande do Sul: Canela (Barragem dos Bugres), 5 ơ, 6 ㅇ, 13-14.XII.1999, A. B. Bonaldo col. (MCN 31880); São Francisco de Paula, O, 22.IX.1974, A. A. Lise col. (MCN 2303); (Passo dos Bugres), 2 O , 4 +, 04.XI.1998, A. B. Bonaldo col. (MCN 30341).

Distribuição. México (Tabasco e Chiapas), Guatemala (Izabal), Costa Rica (Heredia, Limon e Putarenas), Panamá (Panamá), Colômbia (Meta, Choco e Valle), Equador (Morona-Santiago), Peru (Montenegro) e Brasil (Rio de Janeiro e Rio Grande do Sul).

\section{Naatlo splendida (Taczanowski, 1873)}

Theridium splendidum TACZANOWSKI, 1873:5.

Theridiosoma splendida; KeYserLInG, 1884:221, pr. 10, fig. 134; ARCHER, 1953:12, fig. 20.

Naatlo splendida; CodDington, 1986:47, figs. 73, $84-90$ (descrição do macho).

Material examinado. BRASIL, Pará: Santa Isabel $(15 \mathrm{Km}$ Sul), O', 14.II.1990, A. B. Bonaldo col. (MCN 32557); Rio Grande do Sul: Derrubadas (Parque Estadual do Turvo), ơ, 27.X.2003, R. Ott col., pitfall trap (MCN 38523).

Distribuição. Colômbia (Meta), Guiana Francesa (Guyana), Peru (Ucayali e Huánuco), Bolívia (Beni) e Brasil (Amazonas, Amapá, Pará e Rio Grande do Sul).

\section{Ogulnius obtectus O. P.-Cambridge, 1882}

Ogulnius obtectus O. P.-CAMBridge, 1882:433, pr. 30, figs. 9, 9ad; Simon, 1895:919, fig. 984; Roewer, 1942:968; Bonnet, 1956:1706; Brignoli, 1981:12, fig. 2; Coddington, 1986:55, figs. 107-119 (descrição do macho).

Material examinado. BRASIL, Amazonas: Manaus (Reserva Florestal Adolpho Ducke), +, 26.VII.1973, L. P. Albuquerque col. (MCN 32596).

Distribuição. Colômbia (Magdalena e Putumayo), Peru (Ucayali e Cuzco) e Brasil (Amazonas).

Agradecimentos. À curadora do MCN, Erica H. Buckup, pelo acesso ao material e à bibliografia. Ao Dr. Antonio D. Brescovit (Instituto Butantan), pelo envio de bibliografia. Ao Luciano de A. Moura (MCN), à Maria Aparecida de L. Marques (MCN) e aos revisores anônimos, pelas sugestões apresentadas. À direção do $\mathrm{MCN}$, pelo uso das dependências e equipamentos. Ao CNPq, pela concessão da bolsa de mestrado a E. N. L. Rodrigues (proc. 131628/2005-4).

\section{REFERÊNCIAS BIBLIOGRÁFICAS}

ARCHER, A. F. 1953. Studies in the orbweaving spiders (Argiopidae), 3. American Museum Novitates 1622:1-27.

Bonnet, P. 1956. Bibliographia araneorum; analyse méthodique de toute la littérature araneologique jusqu'en 1939. Toulouse, Douladore. v.2, part 2, p.919-1926.

1959. Bibliographia araneorum; analyse méthodique de toute la littérature araneologique jusqu'en 1939. Toulouse, Douladore. v.2, part 5, p.4231-5058.

Brignoli, P. M. 1981. Spiders from the Philippines IV. A new Ogulnius and notes on some other Oriental and Japanese Theridiosomatidae (Araneae). Acta Arachnologica 30:9-19.

Coddington, J. A. 1986. The genera of the spider family Theridiosomatidae. Smithsonian Contributions to Zoology 422:1-96.

1990. Ontogeny and homology in the male palpus of orbweaving spiders and their relatives, with comments on phylogeny (Araneoclada: Araneoidea, Deinopoidea). Smithsonian Contributions to Zoology 496:1-52.

Keyserling, E. 1884. Die Spinnen Amerikas, Theridiidae. Nürnberg, Bauer \& Raspe. Band, 2, Hälfte 1. 222p.

Pickard-Cambridge, F. O. 1902. Arachnida - Araneidea and Opiliones. Biologia Centrali-Americana, Zoology. London, British Museum (Natural History). v. 2, p.313-424.

Pickard-Cambridge, O. 1882. On new genera and species of Araneidea. Proceedings of the Zoological Society of London 1882:423-442.

1896. Arachnida. Araneida. Biologia CentraliAmericana, Zoology. London, British Museum (Natural History). v.1, p.161-224.

Platnick, N. I. 2005. The world spider catalog, version 5.5 American Museum of Natural History. Disponível em: <http:/ /research.amnh.org/entomology/spiders/catalog/index.html>. Acesso em: 05.2005.

Rodrigues, E. N. L. \& Otт, R. 2005. Nova espécie de Theridiosoma (Araneae, Theridiosomatidae) do sul do Brasil. Iheringia, Série Zoologia, 95(1):79-81.

Roewer, C. F. 1942. Katalog der Araneae von 1758 bis 1940. Bremen, Kommissions-Verlag von "Natura". v. 1, p.1-1040.

Simon, E. 1895. Histoire Naturelle des Araignées. Paris, Librarie Encyclopédique de Roret. v.1, fasc. 4, p.7611084.

1903. Histoire Naturelle des Araignées. Paris, Librarie Encyclopédique de Roret. v.2, fasc. 4, p.669-1080.

TACZANOwski, L. 1873. Les aranéides de la Guyane Française. Horae Societas Entomologicae Rossicae 10:1-115. 